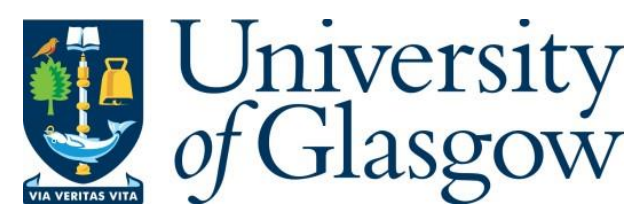

Gallacher, K. et al. (2017) Germanium-on-silicon Waveguides for Mid-infrared Photonic Sensing Chips. In: 2017 IEEE 14th International Conference on Group IV Photonics (GFP), Berlin, Germany, 23-25 Aug 2017, pp. 63-64. ISBN 9781509065684.

There may be differences between this version and the published version. You are advised to consult the publisher's version if you wish to cite from it.

http://eprints.gla.ac.uk/152541/

Deposited on: 27 November 2017

Enlighten - Research publications by members of the University of Glasgow http://eprints.gla.ac.uk 


\title{
Germanium-on-Silicon Waveguides for Mid-Infrared Photonic Sensing Chips
}

\author{
Kevin Gallacher ${ }^{1}$, Leonetta Baldassarre ${ }^{2}$, Ross W. Millar ${ }^{1}$, Alessia Sorgi ${ }^{2}$, Valeria Giliberti ${ }^{3}$, Jacopo Frigerio ${ }^{4}$, \\ Giovanni Isella ${ }^{4}$, Isabella Figliolia ${ }^{5}$, Paolo Biagioni ${ }^{5}$, Michele Ortolani ${ }^{2}$, and Douglas J. Paul ${ }^{1}$ \\ 'School of Engineering, University of Glasgow, Glasgow, G12 8LT, U.K. \\ ${ }^{2}$ Department of Physics, Sapienza University of Rome, Rome, 00185 Italy \\ ${ }^{3}$ Center for Life Nanosciences, Istituto Italiano di Tecnologia, Rome, 00185 Italy \\ ${ }^{4}$ L-NESS, Dipartimento di Fisica del Politecnico di Milano, Como, 22100 Italy \\ ${ }^{5}$ Dipartimento di Fisica, Politecnico di Milano, Milano, 20133 Italy \\ Email: Douglas.Paul@glasgow.ac.uk
}

\begin{abstract}
Germanium-on-silicon rib waveguides are modelled, fabricated and characterized with a novel near-field infrared spectroscopy technique that allows on-chip investigation of the waveguide losses at $5.8 \mu \mathrm{m}$ wavelength.
\end{abstract}

\section{INTRODUCTION}

Silicon-on-insulator (SOI) has been extensively researched for complex on-chip photonic integrated circuits that operate at telecommunication wavelengths. However, for longer wavelength applications that exist in the mid-infrared (MIR), such as sensing of molecular vibrations in the 3-15 $\mu \mathrm{m}$ wavelength range requires a different waveguide platform [1]. This is due to $\mathrm{SiO}_{2}$, which is not optically transparent and therefore causes large waveguide losses. An alternative platform that has been suggested is Ge-on-Si. This is an excellent candidate since both $\mathrm{Si}$ and $\mathrm{Ge}$ are broadly transparent over the full MIR. In addition, due to the refractive index contrast between $\mathrm{Ge}$ and $\mathrm{Si}$ it is possible to form photonic waveguides. Whilst, Ge-on-Si waveguides have been proposed there has only been a few demonstrations [2-3]. In this paper, low loss Ge-on-Si rib waveguides operating at $5.8 \mu \mathrm{m}$ wavelength are demonstrated. These are characterized by a novel measurement technique based on an atomic force microscope (AFM-IR) infrared spectroscopy system.

\section{MODELLING}

Modelling of the supported modes of Ge-on-Si waveguides was performed by a finite difference eigenmode solver. The selected waveguide structure investigated was a rib since it less sensitive to sidewall roughness compared to a ridge. In addition, the rib waveguide only requires a partial etch to form the guiding region, which therefore relaxes fabrication. Figure 1 shows the effective index for the optical modes supported in a Ge-on-Si rib waveguide with a total Ge thickness of $2 \mu \mathrm{m}$ and a slab thickness of $1 \mu \mathrm{m}$. It is clear that single mode condition is obtained for waveguide widths smaller than $4.25 \mu \mathrm{m}$. To couple into the rib waveguide, grating couplers were modelled by FDTD and these were optimized for $5.8 \mu \mathrm{m}$ wavelength.

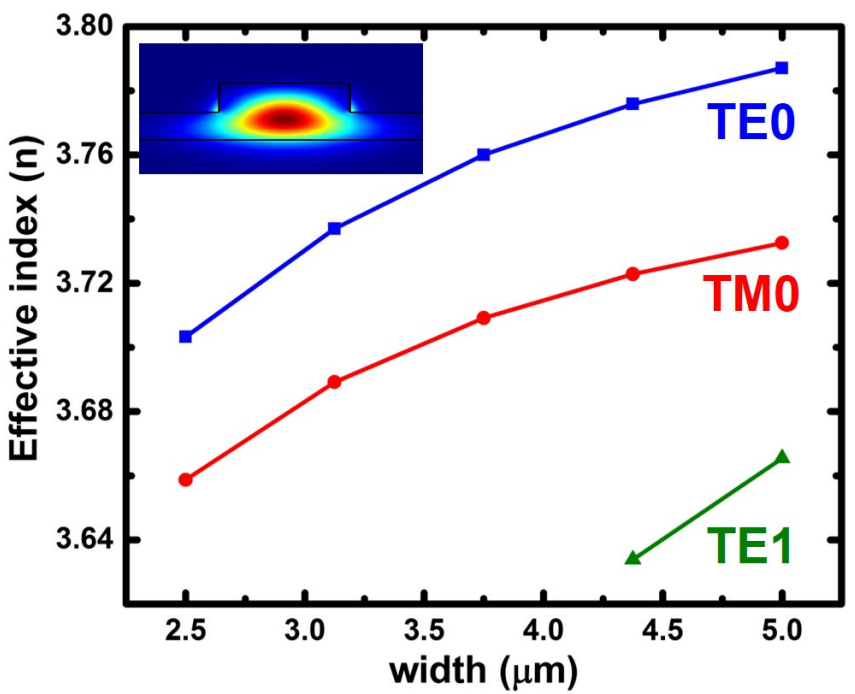

Figure 1. Effective index for the optical modes supported in a Ge-on-Si rib waveguide versus width operating at $5.8 \mu \mathrm{m}$ wavelength. The inset shows the electromagnetic field profile for the fundamental TE mode.

\section{FABRICATION}

The Ge required for waveguide fabrication was grown epitaxially by low-energy plasma-enhanced chemical vapour deposition on a high resistivity (100) Si substrate [4]. After growth, the Ge was cyclic annealed at high temperature to reduce the threading dislocation density to $\sim 10^{7} \mathrm{~cm}^{-2}$. The rib waveguides were subsequently fabricated by patterning HSQ by electron-beam lithography. The grating coupler and rib waveguides were dry etched at the same time using a mixed gas process $\left(\mathrm{SF}_{6} / \mathrm{C}_{4} \mathrm{~F}_{8}\right)$. This etch process has been optimized to be highly anisotropic and to produce ultra-smooth sidewalls [5]. Next a trench is etched through the remaining Ge slab at the output facet of the rib waveguide, before an SU8 polymer block is subsequently patterned and cured. This etched trench is essential to ensure high coupling $(\sim 60 \%)$ from the rib waveguide into the SU8 polymer block. Otherwise the Ge slab acts as a planar waveguide and prevents most of the light reaching the SU8. 

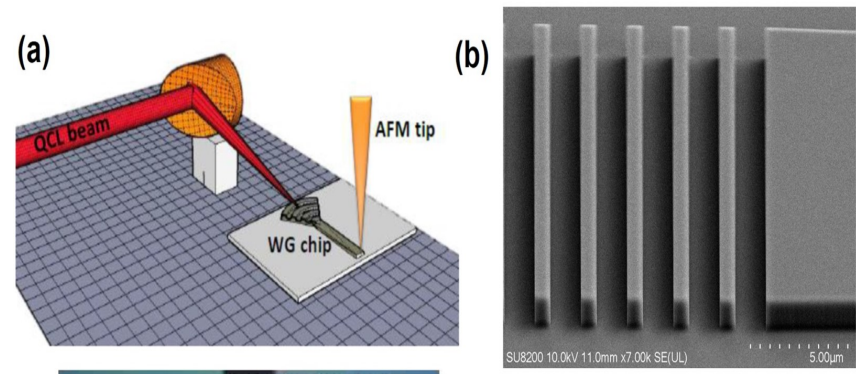

(c)

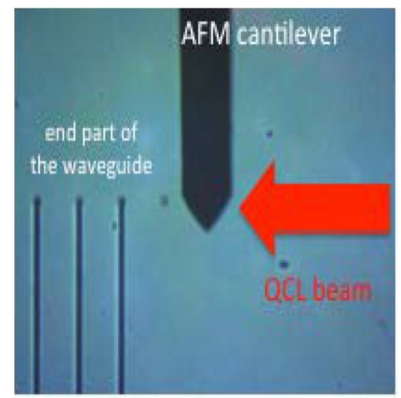

(d)

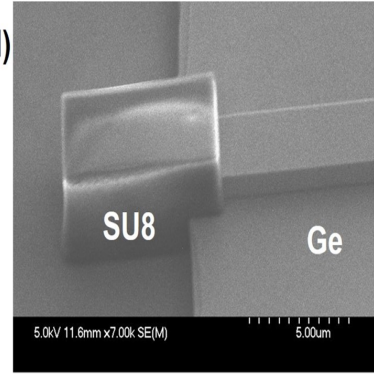

Figure 2. (a) Schematic diagram of the AFM-IR technique to measure waveguide loss of Ge-on-Si rib waveguides. The QCL beam is focused onto the grating coupler and the light coupled to the end of the rib waveguide causes thermal expansion of a SU8 block that is measured by the AFM tip. (b) SEM image of a fabricated grating coupler. (c) Optical microscope image of the AFM tip and the rib waveguides. (d) SEM image of the SU8 polymer block patterned at the rib output facet.

\section{CHARACTERIZATION}

The characterization of the fabricated rib waveguides was performed by using an AFM-IR system (NanoIR2). AFM-IR works by using a MIR source such as a quantum cascade laser (QCL) to induce vibrational absorption and thus thermal expansion into a sample of interest, which is then measured as a cantilever deflection by the AFM. The QCL is normally focused to the same point as the AFM tip to probe the sample but for the waveguide characterization setup, the QCL beam is purposely misaligned and positioned onto the grating coupler, whilst the AFM tip is positioned at the output of the rib waveguide on top of the SU8 block (see Figure 2). SU8 polymer works well since it has very strong absorption in the MIR $\left(>4000 \mathrm{~cm}^{-1}\right)$. Hence, the SU8 and AFM act as the detector for the system. It has been verified that the AFM-IR signal is linear with the intensity of light impinging the SU8 block. Therefore, waveguide losses can be measured by changing the waveguide length and measuring the change in expansion. The output of the Ge-on-Si rib waveguide where the SU8 block is located can be seen in more detail in Figure 2. From measurements performed on waveguides with varying lengths it is clear that very strong Fabry-Perot fringes are being observed. After some investigation, it was established that a cavity is forming between the end facet of the waveguide and the grating that is also acting as a Bragg reflector. Figure 3 shows the behavior of the interference fringes for three different taper lengths and four waveguide lengths. The visibility of the fringes increases for longer tapers as there is better coupling from the multi-mode grating to the single mode waveguide region. The fringe period is compatible with the distance between the grating coupler and the output facet of the waveguide. Since clear interference fringes are observed the waveguide transmission loss can be calculated directly by the Fabry-Perot fringe contrast method. From the ratio between the fringe peak and the minimum, this calculation leads to a propagation loss of $\sim 2 \mathrm{~dB} / \mathrm{cm}$.

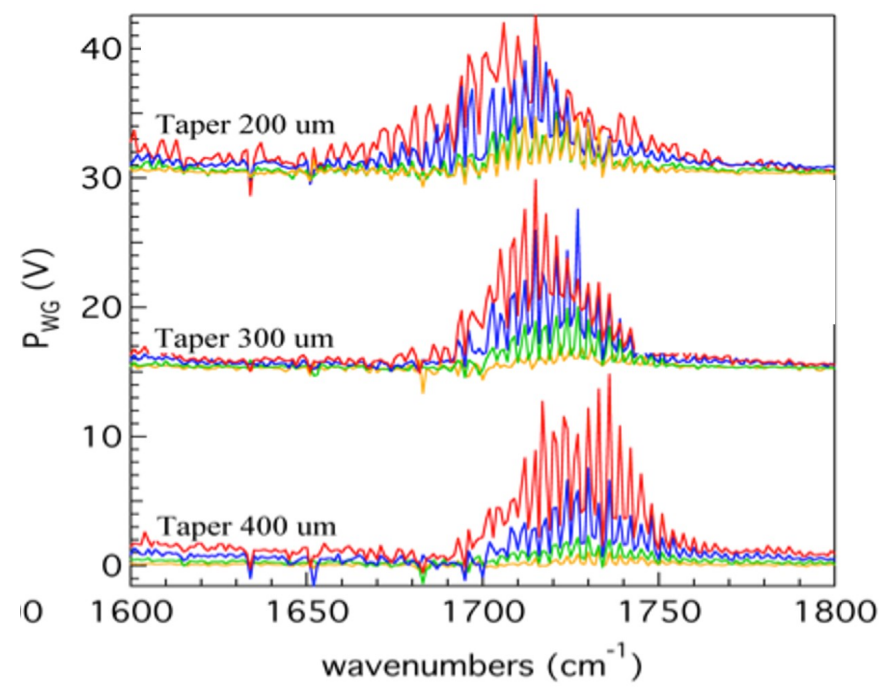

Figure 3. The AFM deflection voltage caused by light reaching the SU8 block from the Ge-on-Si rib waveguides. The plot shows the deflection for different waveguide lengths with different inverse linear tapers. The fringe spacing is changing for varying waveguide lengths.

\section{CONCLUSION}

Ge-on-Si rib waveguides have been modelled, fabricated and then characterized by a novel AFM-IR system. The waveguide losses have been calculated to be $\sim 2 \mathrm{~dB} / \mathrm{cm}$. This represent an exciting perspective for MIR integrated silicon photonic sensing chips.

\section{REFERENCES}

[1] Baldassarre L., et al., "Mid-infrared plasmon-enhanced with germanium antennas on silicon substrates", Nano Letters, 15,7225 (2015).

[2] B. Troia, et al., "Germanium-on-silicon Vernier-effect photonic microcavities for the mid-infrared," Opt. Lett. 41, 610-613 (2016).

[3] Y. C. Chang, et al., "Cocaine detection by a mid-infrared waveguide integrated with a microfluidic chip", Lab on a Chip, 12, 3020 (2012).

[4] Chrastina, D., et al., "Thin relaxed SiGe virtual substrates grown by lowenergy plasma-enhanced chemical vapor deposition. Journal of Crystal Growth, 2005. 281(2-4): p. 281-289

[5] M. M. Mirza, et al., "Nanofabrication of high aspect ratio ( 50:1) sub-10 $\mathrm{nm}$ silicon nanowires using inductively coupled plasma etching," J. Vac. Sci.Technol. B, Microelectron. Nanometer Struct., Sep. 2012 